\title{
LATE STAGES OF STELLAR EVOLUTION
}

\author{
P. THEJLL \\ Niels Bohr Institute, Blegdamsvej 17 \\ DK-2100 Copenhagen $\varnothing$, Denmark
}

\begin{abstract}
A review is given of the use of high-accuracy astrometry on research on white dwarfs and the hot subdwarfs and central stars of planetary nebulae (CSPN). Predictions are made about the expected impact of HIPPARCOS, and the possible impacts of GAIA and ROEMER. Discovery of large numbers of new white dwarfs is expected, and, for the more distant hot subdwarfs and CSPN, important refinements of our current understanding of these objects. For white dwarfs independent values of mass and radius may be accurate enough to allow new understanding of the internal composition.
\end{abstract}

\section{Introduction}

Recently proposed astrometric space observatories (the HIPPARCOS-like but much more sensitive ROEMER, Bastian et al. 1993, and the astrometric interferometer GAIA, Lindegren et al. 1993) have the potential for drastically increasing the precision with which parallaxes and proper motions could be measured for faint stars. What are the predictable consequences of such data for research into White Dwarfs (WD), the Planetary Nebulae (PN) phase and the hot subdwarfs near the end of the horizontal branch (HB)?

White dwarf research provides testable predictions of stellar evolution as well as furnishing test-cases for many areas of physics, such as atomic physics (light-absorption under very high pressure and strong magnetic fields), quantum mechanics (the WD mass-radius relation) and general relativity (gravitational red-shift). Cosmological questions can be addressed with the WDs as they can measure the age of star-forming regions. As WD stars represent the final fate of $90 \%$ of all stars they are a key part of stud- 
ies on stellar evolution: All the processes that stars undergo during their evolution must have the net effect of producing WDs with the observed properties.

Hot subdwarf research is of interest to investigations of stellar mass-loss, as these stars may represent the extreme case of near-complete envelope loss during single star evolution. This is the key to understanding the chemical evolution of a galaxy or cluster of stars over several stellar generations. Hot subdwarfs may furthermore explain the observed ultraviolet upturn in the flux from metal rich galaxies (Greggio\&Renzini 1990) and are an important source of ionizing radiation at high galactic latitude (de Boer 1985).

\section{White Dwarf research topics and the impact of astrometry}

\subsection{THE WD MASS}

The mass-loss on the giant branches, and the Helium flash which terminates red giant branch (RGB) evolution determine the mass that WDs have. The best measurement of the mean WD mass is that by Bergeron et al. 1992 (BSL92 hereafter), who found $\left\langle M_{\mathrm{WD}}\right\rangle=0.53 \pm 0.13 \mathrm{M}_{\odot}$ in good agreement with a typical core mass on the RGB of close to $0.5 \mathrm{M}_{\odot}$ for stars with initial mass below about $2 \mathrm{M}_{\odot}$ (Sweigart\&Gross 1978). For stars of higher initial mass the evolution is much more rapid and the mass loss is also important during other evolutionary phases.

\subsection{DETERMINING WD MASSES}

Standard methods of mass determination for WDs have recently been discussed by BSL92. Briefly summarized the methods relevant here are: 1) Keplers third law, 2) Using parallaxes and a mass-radius relationship, 3) Spectroscopic gravities and a mass-radius relationship, and 4) Spectroscopic gravities and parallax. Precise parallaxes have an immediate impact on all these methods. Method 1 is limited by the availability of suitable systems to a very small number: 40 Eri B (Heintz 1974) and Sirius B (Gatewood\& Gatewood 1978) provide the only really good data points. Other binary systems have such long periods that changes in the positions of the stars are unobservable with present technology. Stein $2051 \mathrm{~B}$ is an example of a star that would be analyzable if observed with $\mu$ as accuracy. Method 2 uses an estimate of the effective temperature and the absolute magnitude, to give a radius which is then used with a theoretical mass-radius relationship (Hamada\&Salpeter 1961) to yield the mass. Methods 3 and 4 first derive the gravity, $g$, from spectroscopic analysis and then (method 3 ) derives the mass from a mass-radius relationship or (Method 4) derives the mass from $4 \pi H_{\nu} R^{2}=f_{\nu} D^{2}$ where $D$ is known from the parallax, $H_{\nu}$ is the 
Eddington flux at some specific wavelength for the model spectrum that best fits the spectral features of the real star, $f_{\nu}$ is the observed flux at the same wavelength and $R$ is the radius determined from $g=\frac{G M}{R^{2}}$.

Accurate masses for WD's would allow a reopening of the question of whether the hydrogen-rich (DA) and helium-rich (DB) white dwarfs have the same mean mass (Shipman 1979). As that argument is discussed on the basis of statistics, increasing the number of known DB would be of great help.

\subsection{THE WD MASS-RADIUS RELATION}

A striking property of WDs is that their internal structure is governed by quantum mechanics - therefore their bulk properties, such as the radius for a given mass, depend on quantum mechanics. The mass-radius relationship is mostly used in the predictive sense, illustrating confidence in the relation: Given a mass (or radius) for a WD, the radius (or mass) is predicted. Testing the M-R relation is limited by the availability of suitable systems. At present only the WD's Sirius B and 40 Eri B and to some extent Stein $2051 B$ 'test' the M-R relation of Chandrasekhar. There is no real doubt that the relationship is basically valid but the issue is in fact only addressed by these few systems. If 1 in a thousand WD's is a suitable binary system then we may expect several times more test-cases if the proposed observatories are launched. The ultimate allure of the M-R relation is that it depends on chemical composition - with accurate enough independent masses and radii one could find the internal composition of WD's.

\subsection{NEW WD'S WILL BE FOUND}

There are several ways in which the proposed space observatories could help increase the number of analyzable binary systems with WDs, as well as WDs in general: First of all, frequent photometric monitoring of systems containing WDs would help reveal eclipsing systems - at present there are no concerted efforts to keep an eye on all known WD's (at least 2000 are known-McCook\&Sion 1987) so it is not known whether some subset of the known WD's are undergoing eclipses or not. Secondly, the greatly increased accuracy of the proposed observatories will make it possible to study the motion of stars in long period binary systems that have periods that are too long for the present accuracy to give meaningful data (e.g. Stein 2051B). Common proper motion pairs will also be detected for input to detailed binary system studies.

Many WDs have been found in faint large proper motion surveys and the proposed astrometric observatories could reveal many new WDs. As about 40,000 WD's are predicted to be closer than $100 \mathrm{pc}$, the number of WD's 
available for study would increase by an order of magnitude or more above what is currently available. To detect previously unknown stars the mission must either operate in a sky-survey mode or operate from prepared input catalogs that contain all stars, as opposed to stars from existing catalogs. The proper motions that have been measured fall in the range from 0 to 4 arcseconds/yr with half above 300 mas/yr. Improved hardware could set lower and lower limits to the detectable proper motion $(0.1 \mathrm{mas} / \mathrm{yr}$ below $\mathrm{V}=11$ and 1.5 mas at $\mathrm{V}=17$ for ROEMER) but procedures must be adapted to searching for fast movers in fields that will be increasingly crowded as the limiting magnitude is pushed higher and higher. Hot WDs are UV bright and any astrometric mission with UV sensitive detectors would be particularly useful for target-identification.

\subsection{AGE OF THE GALAXY FROM WD COOLING}

The age of the star forming region of the Galaxy can been estimated by measuring the luminosity function of cooling WDs (Liebert et al. 1988, and Wood 1992).

White dwarfs cool at a calculable rate that depends mainly on the present temperature of the star, but also on the internal structure and composition. The coolest WD's in the solar neighborhood set limits on the age of the disk. So far, the age measured for the disk from the intrinsically faintest WD's is rather low (9 - $10 \mathrm{Gyr})$ when compared to Globular Cluster (GC) ages (12 - $18 \mathrm{Gyr})$. As the detection and counting of WDs is fairly simple compared to GC isochrone fits, the WD age-determination has raised interesting questions regarding both the methods used in isochrone fitting, the relative timing of stellar evolution in various parts of the Galaxy and the theory of WD cooling.

The possibility for deep surveys of WD's is exciting in the context of gathering non-biased data. With the ROEMER basically all hot WDs (with cooling times of the order $1 \mathrm{Gyr}$ - easily selectable from their color) could be observed out to 100 parsec with an accuracy better than 1.5 mas. This is presently possible with HIPPARCOS only for stars inside the closest 10 - 15 parsec.

\section{Progenitors of WD's}

By measuring distances one can calculate space densities for stars in different but connected stages of evolution and test stellar evolution theories by comparing the evolution rates. Most WDs are formed via the PN route, while a minority is formed by those stars that miss the Asymptotic Giant Branch phase and go directly from the blue HB to the WD cooling sequence. As some stars - notably the helium-rich subdwarf O stars (sdO) 
and the helium-rich DB WDs - are chemically peculiar, it has been suggested that such sub-classes are linked. A direct test of this suggestion could be performed if accurate rates of evolution were known for both classes of objects.

At present the space density of helium rich WDs is only indirectly known: the fraction of DB's among WD's is known and the total DA space density is known (Fleming et al. 1986), but no direct measurement of the DB space density exists for several reasons: DB's are rare, so large numbers are needed before a meaningful statistic can be measured, and the main method available for DB distance determination has so far been spectrophotometric: There are only parallaxes for $9 \%$ of the DB's in the McCook\&Sion catalog and models for DB's have only recently become reliable (see Thejll et al. (1991) for a discussion of this point).

The space density of the helium rich sdO stars that sit in the highluminosity area above the blue tip of the $\mathrm{HB}$ is not well known as the only method ever attempted for sdO distance determinations is unreliable: Dreizler et al. 1990 and Thejll et al. 1994 analyze the same stars with different models and find significantly different results. Trigonometric parallaxes for the sdO would give masses which, by comparison to stellar evolution predictions, could solve the problem which is of interest to the development of new radiative transfer methods for hot stars. So far, only one parallax is known for a hot sdO - the 10'th magnitude Feige 34 has a $50 \%$ accurate parallax in the revised Yale catalog (van Altena et al. , 1991). The stars are mostly above the galactic plane and most are far away and have not been tempting targets for astrometrists. sdO masses vary - all known values are derived from binary systems (Ritter 1990), more of which are needed.

The space density of central stars of planetary nebulae (CSPN) is not well known, but methods, based on the illuminating effect of the central star on the nebula, are available that are not applicable to the sdO case. The Zanstra method allows estimation (although somewhat uncertainly) of the luminosity of the CSPN and this gives distance estimates. Direct parallaxes are needed for determining the space density, and for testing the accuracy of the Zanstra method so that it can be used with greater confidence for far-away nebulae outside the range of parallax measurements.

\section{Discussion of requirements for astrometric missions}

What are the characteristics of an experiment that would accurately yield space densities and scale heights for WDs and hot subdwarfs?

For WDs the answer depends on the absolute magnitude (and hence age) one wants to study. The wide range of absolute magnitudes present strongly determines what can be done with the proposed technology. Hot WDs (say 


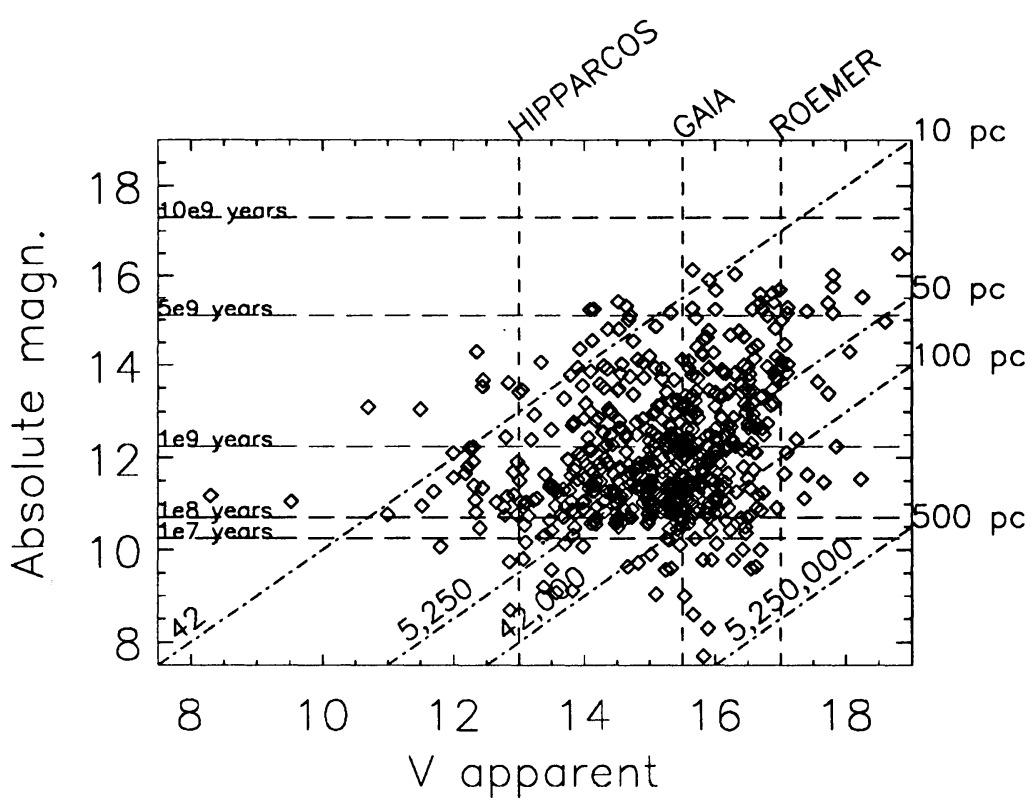

Figure 1. 575 WD's with known V magnitude and absolute magnitude plotted against each other, with horizontal lines showing the cooling times in years, diagonal lines showing the distances and vertical lines showing the limiting magnitudes for HIPPARCOS, GAIA and ROEMER. At the lower end of each distance-line is a number indicating the number of WD's that are closer than the corresponding distance, based on current measurements of WD space density. Only for the very closest WD's is the presently known sample complete.

at $M_{\mathrm{abs}}=10$ ) are at 500 parsec distance (roughly 2 scale heights) at $\mathrm{V}=19$ while $M_{\mathrm{abs}}=15$ WDs require that one can observe reliably at $\mathrm{V}=23$. None of the suggested missions have this capability, although the ROEMER mission is aiming for a capability of 1.5 mas accurate parallaxes at $\mathrm{V}=17$, which might cover 1 scale height, or a little more, of the hot WD distribution and give $30 \%$ accurate parallaxes at the limit, which is competitive with spectrophotometric distances. GAIA would cover less than a scale height, but proposes to give parallaxes accurate to $20 \mu$ as at $\mathrm{V}=15$, which in the hot WD case means distances accurate to $0.2 \%$ - virtually error free. Cool (old, Pop II) WDs would still be best dealt with spectrophotometrically unless mas accuracy can be provided at $24^{\prime}$ th and $25^{\prime}$ th magnitude. With scale height known, space density could be calculated and then evolution rates would follow using the known cooling rate for WDs.

Direct measurement of the space density of WDs by having complete counts of the stars in the solar vicinity - possible even for the coolest and oldest WDs - seems a preferable method, but is possible only if the large number of WDs undetected nearby the Sun are located and accounted for. 
In figure 1 we see that there should be about 5000 WDs within 50 parsec of the Sun, most of which would be observable by ROEMER and to a lesser extent GAIA.

GAIA's potential importance to WD research lies in the higher accuracy attainable than with ROEMER - but as ROEMER reaches 2 magnitudes fainter the latter may well have the greater impact on WD research where accuracy in parallaxes is important but reaching further into space is the key to arguing against claims of local solar neighborhood bias.

Hot sdB and sdO and the CSPN can be seen much further away than WD's as they have luminosities from 10 to hundreds of solar luminosities. It is therefore hoped that a spectrophotometric distance determination can be performed on the stars in the complete Palomar-Green survey of high galactic latitude blue objects (Green et al. 1986). However, this requires advanced NLTE model atmosphere techniques that have problems in predicting the line profiles consistently in comparisons between different sets of models - particularly for the sdO stars but also, to a lesser extent, for the somewhat cooler hydrogen-rich subdwarf B (sdB) stars.

The sdB probably have the mass of a stripped He core - i.e. $0.5 \mathrm{M}_{\odot}$ but the sdO could in principle have a range of masses from tenths of solar masses to about $2 \mathrm{M}_{\odot}$. The scale height for the sdB has been determined assuming the mass is $0.5 \mathrm{M}_{\odot}$ and it is near 500 parsec (Saffer 1994) and stars as far away as a few kiloparsec have been analyzed, so the requirement of covering a sufficient number of scale heights is met for the sdB.

Spectrophotometric mass-determinations for sdO, coupled to parallax work, relies on the basic equation in section 2.2. Once the modelling problems were resolved one could rely on spectrophotometric values for $g$ and then determine masses: A realistic accuracy in spectroscopically determined gravities is 0.2 or 0.1 in $\log (g)$. Once $\pi$ was known better than $50 \%$ to $25 \%$ one could get masses with accuracies mainly limited by $\Delta \log (g)$.

HIPPARCOS can just manage to yield the required parallax accuracy for a small number of sdO bright enough and the release of data is eagerly awaited. The less problematic sdB will have their problems sorted out by more accurate missions. GAIA can, with $20 \mu$ as accurate parallaxes at $\mathrm{V}=15$ give $3 \%$ accurate distances for nearly half of the presently known hot subdwarfs, and ROEMER could cover all known hot subdwarfs, putting the emphasis on improving the accuracy of spectral modelling methods.

The impact of HIPPARCOS, to some extent, and GAIA and ROEMER in particular, on resolving present problems of NLTE modelling will be great. Once the problems are sorted out, spectrophotometric methods in conjunction with parallaxes will deliver $50 \%$ accurate individual sdO masses and more accurate sdB masses. This is enough to test whether the two groups of stars have a well defined mean mass or not, and whether it is the 
same, which is sought-after information with impact on stellar evolution theory.

\subsection{SUMMARY}

The properties of the proposed observatories are such that there could be important consequences in two broad areas of research in late stages of stellar evolution: more accurate derivation of stellar properties for more stars, and detection of previously unknown stars. The discovery of many new white dwarfs, the significantly more accurate study of which will yield more and better insight into WD masses, radii, spatial distribution and hence the luminosity function, and the age-determination of the stars in the Galaxy is one of the most important potential results. For the hot subdwarfs and CSPNs reliable answers can be expected to questions about the spatial distribution and hence the role played by these stars on the road to the WD's and as tracers of stars with extreme mass-loss. Of great interest will be the many opportunities for testing various methods of measurement and analysis.

Support from the IAU, the Carlsberg foundation, and the NBI is gratefully acknowledged.

\section{References}

Bastian, U. et al. (1993), ROEMER, Proposal for M3

Bergeron, P., et al. (1992), ApJ 394, 228, BSL92

de Boer, K. (1985), A\& 142, 321

Dreizler S. et al. (1990), A\&A 235, 234

Fleming T. et al. (1986), ApJ 308, 176

Gatewood G.D.\& Gatewood C.V. (1978), ApJ 225, 191.

Green R.F. et al. (1986), ApJSS 61, 305

Greggio\&Renzini, A. (1990), ApJ 364, 35

Hamada T.\&Salpeter E.E. (1961), ApJ 134, 683

Heintz W.D. (1974), AJ 79, 819

Liebert J. et al. (1988), ApJ 332, 891

Lindegren L., et al. (1993), GAIA, Proposal for an ESA Cornerstone Mission

McCook\&Sion E.M. (1987) ApJSS 65, 603

Ritter H. (1990), AASS 85, 1179

Saffer R. (1994), in White Dwarfs, proceedings of the 9'th European WD Workshop.

Shipman, H.L. (1979), ApJ 228, 240

Sweigart A.V.\&Gross P.G. (1978), ApJSS 36, 405

Thejll P.A. et al. (1991), ApJ 370, 355

Thejll P.A. et al. (1994), ApJ, in press

Wood M. (1992), ApJ 386, 539

van Altena W.F. et al. (1991), Gen. Cat. of Trig. Par.: Prel. Ver. 